\title{
Dopamine in Immunoregulation
}

\author{
Istvan Berczi and Toshihiko Katafuchi \\ Editors in Chief
}

In this issue Toth et al. [1] discusses the role of peripheral and brain derived dopamine (DA) in immune regulation. Here we consider briefly the possible way by which hormones and other central regulatory factors act peripherally in vivo.

During the early years of the nineteenth centaury Endocrinologists recognized first when searching for the origin of hormones, that "everything is made everywhere". This rule was true for all hormones and neurotransmitters that have been examined. This phenomenon has been re-discovered since that time over and over again.

DA exerts an inhibitory effect on prolactin (PRL) and growth hormone $(\mathrm{GH})$ secretion. This effect is very powerful. In rats treatment with bromocriptine (a DA agonist drug), inhibited completely the adaptive immune function (ADIM) [2]. The rule in the Neuroimmune Supersystem [3] is that mediators are shared: so classical hormones and neuropeptides are made in the immune system and everywhere else, lymphokines were discovered in the immune system first, but then were found in many other tissues and cells so their name was changed to cytokines, which implies tissue hormones. Sharing makes communication instantaneously possible [3]. Dopamine is no exception to this rule. It is made in the central nervous system (CNS) but also in various cell types in the immune system, and in many other tissue and organs in the body. In short, dopamine is made everywhere and acts everywhere [1].

One may argue that dopamine is a powerful immunoregulator, it can act centrally by inhibiting PRL secretion, and also acts peripherally by paracrine/autocrine $(\mathrm{P} / \mathrm{A})$ regulation. Yes indeed, this is very important from the point of usefulness. But how the central and peripheral mechanisms work under homeostatic conditions? There are myriads of mediators produced centrally, peripherally, and receptors are expressed and may be activated everywhere.
This may lead to a very chaotic situation, signals are delivered everywhere; cells could go crazy under such bombarding. It was proposed that membrane bound receptors will cap on one pole of the cell surface after activation by specific ligands. Such receptors are phosphorylated by protein kinases and de-phoshorylated by phosphatases, which happens competitively at the same time. If phosphorylation is stronger the cell will be activated, if de-phosphorylation is stronger, the cells will be inhibited [4].

But there are many other mediators that do not use this signal transmission pathway. Alternate mechanisms are used by some membrane receptors. Also, there are cytoplasmic and nuclear receptors, which definitely would not be controlled by this kind of regulation [5]. It is important that every regulatory circuit has positive and negative signals and the signals will balance out physiologically, possibly under CNS control. The fact is that in spite of the countless mediators, receptors and signaling, the organism remains normal, all functions are in harmony and in homeostasis.

The classical answer is that the CNS controls everything, so the peripheral mechanisms are kept under control by the CNS. Indeed we observed that PRL production by lymphocytes was regulated by the pituitary gland [6]. This would suggest that the CNS might exert a regulatory influence on all regulatory circuits under normal, homeostatic conditions. Could DA of CNS origin signal the immune system or other systems, tissues directly? The answer is yes. Dopaminergic nerves deliver DA to the tissues for local use. Apparently the $\mathrm{CNS}$ is in control here again. So it seems that during homeostasis, the local consumption of DA may be CNS regulated [1].

During homeostasis, cytokines are at minimal or undetectable level in the serum. During acute febrile illness cytokines of innate immunity (INIM) origin [e.g. interleukin - (IL1)-beta, IL-6, and tumor 
necrosis factor - (TNF)-alpha] will rise. Some other cytokines also participate in later phases. This cytokine response initiates the neuroendocrine-, metabolic- and immune alterations associated with acute illness. The brain, the HPA axis, the sympathetic and parasympathetic nervous systems, liver and bone marrow are activated. In the rest of the body catabolism prevails. The sick animal/patient uses it own nutrients and energy resources, as no other sources are capable to satisfy the tremendous demand by the acute immune/inflammatory response [6].

But why a CNS mediator has to be produced locally and set up P/A type regulatory units locally. Because the mediators are shared, DA made centrally could be used locally if necessary. The local circuits seem to be redundant. During homeostasis, tissue cytokines are at minimal or undetectable levels in the serum. This suggests that the P/A circuits are local, not systemic and may perform special local functions. One may suggest that the local circuits are so weak that they are easily overpowered by the centrally produced systemic hormones, neuropeptides, which are well detectable in the serum. It follows that the central regulators at physiological concentrations overload the local circuits and thus dominate them. This would be a very simple principle of regulation, which is also used in Pharmacology, where even higher doses of hormones would overload central physiological circuits and thus dominate/regulate them.

But then why the local circuits are there? Let us suppose that systemic DA is not available as a consequence of injury or disease. In this situation the local regulatory circuits could provide the vital signals for the tissues and cells so the damage to peripheral sites will be moderated or even prevented.

INIM is always with us, protects, and heals us. It prevents disasters and disease in general, provides cytokines, hormones and other mediators, including DA [1]. Apparently tissue viability must be important for the INIM system to work. For instance the bone marrow could not be stimulated by INIM if no cells are viable to stimulate [7]. We suggest that the function of local P/A circuits is to maintain tissue viability in emergency situations, when no other regulators are available. In order to achieve recovery and healing from disease, tissue reactivity is of fundamental importance. Without response from targeted tissues, the INIM system could not initiate healing and recovery. It is apparent that a dead organism can never be healed! Thus the local P/A circuits are the key to healing and recovery. We could not recover from severe debilitating disease without these local regulatory circuits in our tissues.

In clinical practice it is commonly known that the organs and tissues of the body always survive clinical death. The time limit of revival is around 5 minutes if death occurs at about room temperature. It the case of hypothermic death revival is possible for a couple of hours or so. The science of Cryobiology [8] deals with the medical and other application of hypothermia and freezing. Tissue culture is another discipline that deals with growing cells and tissues in vitro from animals and man [9]. These disciplines owe their existence to the ability of P/A circuits to preserve tissue viability and reactivity beyond clinical death for extended periods if handled correctly, or under proper culture conditions. Clearly P/A circuits hold the possibility of resurrection after clinical death.

\section{REFERENCES}

[1] Tóth BE, Vecsernyés M, Zelles T, Kádár K, Nagy GM. Role of peripheral and brain-derived dopamine (DA) in immune regulation (in this issue).

[2] Nagy E, Berczi I, Wren GE, Asa SL, Kovacs K. Immunomodulation by bromocriptine. Immunopharmacology. 1983; 6: 231-243.

[3] Berczi I. Integration and regulation of higher organisms by the neuroimmune supersystem. Int. J. Integrative Biol. 2007; 1(3): 216-231.

[4] Renard V, Cambiaggi A, Vély F, Bléry M, Olcese L, Olivero $\mathrm{S}$, Bouchet M, Vivier E. Transduction of cytotoxic signals in natural killer cells: a general model of fine tuning between activatory and inhibitory pathways in lymphocytes. Immunol. Rev. 1997; 155: 205-221.

[5] Guyton and hall Guyton and Hall. Textbook of Medical Physiology 12th edition 2011 Saunders Elsevier, Philadelphia, USA.

[6] Berczi I, Neuroimmune Biology:. An introduction. In "Neuroimmune Biology Volume 1: New foundation of Biology" I. Berczi I, Gorczynski R, Editors, Elsevier. 2001; 3-45.

[7] Bertok L, Berczi I. Nomenclature and significance of innate/natural immunity and species specific resistance. Advances in Neuroimmune Biology. 2011; 1: 11-24.

[8] Karow AM Jr. Looking back. Cryobiology. 2010; 60(3 Suppl): S2-12.

[9] Pollard JW. Basic cell culture. Methods Mol. Biol. 1990; 5: $1-12$. 